\title{
Higher Gradient Theories and Their Foundations
}

Francesco dell' Isola ${ }^{1}$, Pierre Seppecher ${ }^{2,3,4}$, and Alessandro Della Corte ${ }^{5,6}$

${ }^{1}$ MEMOCS Centre Università dell' Aquila,

DISG Università di Roma La Sapienza,

Rome, Italy

${ }^{2}$ IMATH, Université de Toulon, La Garde,

France

${ }^{3}$ International Research Center M\&MoCS,

L'Aquila, Italy

${ }^{4}$ Institut de Mathématiques, Université de

Toulon, Toulon, France

${ }^{5}$ DIMA, University of Rome La Sapienza,

Rome, Italy

${ }^{6}$ International Research Center M\&MoCS,

University of L'Aquila, L'Aquila, Italy

\section{Synonyms}

Generalized continua; Higher gradient continua; Second gradient continua 


\section{Definition}

Elastic continua whose general deformation energy density depends on second and possibly higher gradients of the displacement.

\section{Introduction}

Continuum mechanics always supplies approximate models for physical systems, in which a more fundamental (possibly discrete or inhomogeneous) microstructure may be somehow neglected. Indeed, Cauchy (or Cauchy-Navier) continuum theory describes efficiently, at a macroscopic level, the behavior of a mechanical system only when the inhomogeneities which the model does not take into account have a characteristic length scale much smaller than the macroscale where phenomena are observed.

Therefore, it is now widely accepted that in some circumstances, it is necessary to add to the placement field some extra kinematical fields, to take into account, at a macroscopic level, some aspects of the mechanical behavior of materials having complex microscopic structures. In the aforementioned direction, a first relevant generalization of Cauchy continuum models was conceived by Eugène and François Cosserat: their efforts were not continued until late in the twentieth century. Cosserat brothers described continuum bodies in which a complete kinematical description of considered continua can be obtained by adding suitable micro-rotation fields. In Cosserat models contact interactions were to be modeled not only by means of surface forces but also by means of surface couples. The conceptual differences between Cauchy-type continuum mechanics and Cosserat-type continuum mechanics were relevant, and the second one cannot be obtained by means of simple modifications of the first one.

As clearly stated already in the works by Germain $(1973 \mathrm{a}, \mathrm{c})$, the principle of virtual work supplies a suitable tool for extending the Cauchy-Navier format of continuum mechanics when it has to be generalized to include the so-called generalized or microstructured continua. This principle has been successfully used, for instance, in the classical works Green and Rivlin (1964a, b, c, 1965), Mindlin (1962, 1963, 1965a, b, c), Mindlin and Tiersten (1962), and Mindlin and Eshel (1968), as well as in dell'Isola and Seppecher (2011), Forest et al. (2015), and Forest (1998, 2005, 2006).

Many results are available by now (see, e.g., Pideri and Seppecher 1997; Alibert et al. 2003; Seppecher et al. 2011; Triantafyllidis and Aifantis 1986) indicating that it is physically needed or mathematically consistent to consider macroscopic continuum models where contact interactions expend work on high order virtual displacement gradients on dividing surfaces. These interactions are exactly those which are called $s$-forces, following Green and Rivlin (see Green and Rivlin 1964a, b, 1965) and the works (Germain 1973a; dell'Isola et al. 2012a). This seems to be an essential common property of all systems that show highly contrasted physical properties at microlevel (see also Chesnais et al. 2012, 2013). On the purely macroscopic point of view, the necessity of considering such interactions has been proven in two very elegant papers by Germain (see Germain 1973a, c) when one wants to consistently consider continuum models in which deformation energy depends on second gradient of displacement (for higher gradients, see dell'Isola et al. 2012a). The conceptual framework introduced by Truesdell and Noll (see Truesdell and Noll 1965) is not general enough for encompassing such models (see, e.g., the difficulties arising in the papers Dunn and Serrin 1985; Dunn 1986 and clarified in dell'Isola and Seppecher 1995).

It should also be remarked that the condition of scale separation mentioned at the beginning is not by itself a sufficient criterion for insuring that Cauchy theory supplies a suitable model: a wellknown example is the case of deformable porous media for which both stress tensor for matrix and pressure for fluid are needed to describe its mechanical state (see, for instance, Collin et al. 2006; Coussy 2004; Sciarra et al. 2007). Another example is given by the case of a periodic fibers 
reinforced elastic medium with high contrast of mechanical properties (see Steigmann and Pipkin 1991; dell'Isola et al. 2016a). The mechanical description of these systems needs in addition to the standard stress tensor a higher-order hyperstress tensor.

To conclude this Introduction, we mention that a list of existing and envisaged applications of generalized continua

includes:

- materials which are able to damp mechanical vibrations by means of a granular microstructure or by transforming mechanical energy into electromagnetic energy via piezoelectric transduction.

- materials which exhibit, at least in some directions, a large ratio between weight and fracture toughness.

- multi-scale fabrics constituted by a beamlike substructure whose deformation energy depends on $n$-th gradient of displacement field and exhibiting nonstandard dispersion effects, possibly including frequency bandgaps.

- materials constituted by nearly inextensible fibers that are able to resist shear and elongation by storing deformation energy in the form of fiber bending energy.

A more comprehensive coverage of applications (and the relative bibliography) is provided and discussed in dell'Isola et al. (2016a).

\section{Interactions Modeled as Work Distributions}

It is evident that describing a force (respectively, a force field) $F$ is equivalent to describing the linear form which, to any test vector $V$ (respectively, test field), associates the expended work $F \cdot V$ (resp. $\int F \cdot V$ ). In this dual view, forces are regarded as distributions in the sense of Laurent Schwartz.

A generally agreed requirement is to admit that the set of all admissible infinitesimal displacement fields for a continuous body $B$ contains the set $\mathscr{D}$ of all test functions (i.e., infinitely differentiable functions having compact support).

In accordance to what is done, e.g., in the works Salençon (2005) and Germain (1973a, b, c), let us recognize that the mechanical interactions applied to an open subbody $D \subset B$ are distributions (in the sense of Schwartz) concentrated on $\bar{D}$, where $\bar{D}$ denotes the topological closure of the set $D$.

Therefore, theorems and definitions of the theory of distributions are relevant also in continuum mechanics. In particular we have to remind that (see Schwartz 1973, pp. 82-103) (i) every distribution having regular (Here "regular" must be understood in the sense of Whitney (see Schwartz 1973, p. 98). This condition is weak enough and all objects considered in this entry verify it) compact support $\bar{D}$ can be represented as the sum of a finite number of derivatives of measures all having their support included in $\bar{D}$; (ii) a distribution is said to have order smaller than or equal to $N$ if one can represent it as the sum of derivatives with order smaller than or equal to $N$ of measures; and (iii) every distribution having support included in a regular embedded submanifold $M$ can be uniquely decomposed as a finite sum of transverse derivatives of extensions of distributions defined on $M$.

As a consequence, any mechanical interaction applied to $D$ has the following structure:

$$
V \in \mathscr{D} \mapsto \sum_{s=0}^{N_{D}} \int\left(\nabla^{s} V\right) \mid d T_{D}^{s},
$$

where $d T_{D}^{i}$ are tensor-valued measures having support in $\bar{D}$ and the symbol | stands for the inner product between tensors.

In order to ensure uniqueness in the representation formulas, it is natural to ask the measures $d T_{D}^{i}$ to respect the same symmetry as $\nabla^{s} V$, that is, to be invariant with respect to any permutation of indices. We call complete symmetry this property and denote $\operatorname{Sym}(X)$ the completely symmetric part of any tensor $X$.

One of the greatest challenges of any continuum mechanics theory is to describe the way in 
which the measures $d T_{D}^{i}$ depend on the shape of $D$. The class of subbodies which are to be considered cannot be limited to domains with smooth boundaries. Indeed tetrahedrons have to belong to this class if we want to follow the trail of Cauchy. Therefore, let us admit subbodies $D$ with boundaries (or Cauchy dividing surface) which are piecewise regular. The topological boundary $\partial D$ is constituted by regular surfaces called faces (their union being denoted $\partial_{2} D$ ), the boundary of which is constituted by regular curves called edges, (their union being denoted $\partial_{1} D$ ) concurring at wedges (their union being denoted $\partial_{0} D$ ). We denote $n_{k}$ the external normal to $D$ on the face $\mathscr{F}_{k}$. On an edge $\mathscr{L}_{j}$ two faces $\mathscr{F}_{k}: k \in\left[\mathscr{L}_{j}\right]$ concur. Hence $\left[\mathscr{L}_{j}\right]$ denotes the pair of subscripts of the faces concurring there. We denote $e_{j}$ a unit vector tangent to the edge $\mathscr{L}_{j}$ and $v_{k}^{j}$ the unit vector orthogonal to the line $\mathscr{L}_{j}$, tangent to the face $\mathscr{F}_{k}$ and external to it. On a wedge $\left\{x_{\ell}\right\}$, a finite number of edges $\mathscr{L}_{j}: j \in\left[x_{\ell}\right]$ concur. Hence $\left[x_{\ell}\right]$ denotes the set of subscripts of the edges concurring there.

The description of the mechanical behavior of a body needs the partition of the mechanical interactions applied to any subbody $D$ into two subclasses, those which are applied inside the body and those which are applied on its frontier:

$$
\begin{aligned}
& \mathfrak{S}^{\mathfrak{i n s}}(D, V)=-\sum_{s=0}^{N_{D}} \int\left(\nabla^{s} V\right) \mid d \tau_{s, D}, \\
& \mathfrak{S}^{\mathfrak{f r o}}(D, V)=\sum_{s=0}^{N_{D}} \int\left(\nabla^{s} V\right) \mid d F_{s, D},
\end{aligned}
$$

where $\tau_{s, D}$ are tensor-valued measures concentrated on $D$, while $d F_{s, D}$ are tensor-valued measures having support in the topological boundary of $D$. At this point the distinction between these two kinds of interactions is completely arbitrary. We emphasize that it has been shown (see Germain 1973a; dell'Isola et al. 2012a) how an expression of type $\mathfrak{S}^{\mathrm{ins}}(D, V)$ can be transformed in an expression of type $\mathfrak{S}^{\mathfrak{f r o}}(D, V)$ while in the works dell'Isola and Seppecher (1997) and dell'Isola et al. (2016b) the converse is shown.
Actually the necessity for mechanicians to divide the mechanical interactions into these subclasses comes from their desire to find constitutive laws for the tensors $\tau$ and $F$ which only involve local quantities. This distinction will be now assumed as granted.

When accepting the point of view by Cauchy, it is the functional $\mathfrak{S}^{\mathfrak{f r o}}$ which characterizes the stress state of the body. When there exists an integer $N=N_{D}$ such that the previous representation holds for all subbodies of the considered body $B$, then it is said that the body $B$ has a stress state of order $N$ in the sense of Cauchy. We deal with measures $d F_{S, D}$ constituted by three parts concentrated on $\partial_{i} D, i=0,1,2$ each one being, respectively, absolutely continuous with respect to the corresponding natural Hausdorff measures:

$$
d F_{s, D}=\mathrm{F}_{s}^{2} d \mathscr{H}_{\mid \partial_{2} D}^{2}+\mathrm{F}_{s}^{1} d \mathscr{H}_{\mid \partial_{1} D}^{1}+\mathrm{F}_{s}^{0} d \mathscr{H}_{\mid \partial_{0} D}^{0}
$$

Moreover we deal with fields $\mathrm{F}_{s}^{i}$ which are smooth tensor fields orthogonal to the manifold where they are applied:

$$
\mathrm{F}_{s}^{i} \perp \partial_{i} D
$$

This is a limitation as in the Schwartz decomposition of distributions concentrated on manifolds dual quantities to tangential components of test functions may appear. Note however that, if these tangent dual quantities are smooth enough to be integrated by parts, they reduce to functions plus dual quantities concentrated on lower-order manifolds. Therefore frontier interactions have the form (The chosen summation bounds may seem restrictive. This is not the case, as one can easily add some extra terms with vanishing densities):

$$
\begin{aligned}
\mathfrak{S}^{\mathfrak{f r o}}(D, V)= & \sum_{s=0}^{N-1} \int_{\partial_{2} D} \mathrm{~F}_{s}^{2} \mid\left(\nabla^{s} V\right)_{\perp} d \mathscr{H}^{2} \\
& +\sum_{s=0}^{N-2} \int_{\partial_{1} D} \mathrm{~F}_{s}^{1} \mid\left(\nabla^{s} V\right)_{\perp} d \mathscr{H}^{1} \\
& +\sum_{s=0}^{N-3} \int_{\partial_{0} D} \mathrm{~F}_{s}^{0} \mid \nabla^{s} V d \mathscr{H}^{0}
\end{aligned}
$$


The tensor fields $\left(\mathrm{F}_{s}^{2}, \mathrm{~F}_{s}^{1}, \mathrm{~F}_{s}^{0}\right)$ which depend on $D$ and on the material particle are naturally completely symmetric and normal to the manifolds where they are applied. They are called the contact $s+1$-forces. For the explicit expression of the generalized forces that appear in second and higher gradient theories, we refer to the entry Generalized contact actions in this Encyclopedia.

One of the essential points of Cauchy approach (see, e.g., Noll 1959 or Fosdick 2011) is the determination of the dependence of the fields $\mathrm{F}_{s}^{i}$ on the (shape of the) subbody $D$. The densities $\mathrm{F}_{s}^{i}$ are assumed to depend in a sufficiently regular way on the position and to depend on the considered subbody only in a local way through its shape: a notion which contains all local geometrical characteristics of the frontier (including its direction). This notion is defined in the work (dell'Isola and Seppecher 1997), where two domains are said to have the same shape if they coincide locally up to a translation.

When accepting the point of view by D'Alembert it is the functional $\mathfrak{S}^{\mathfrak{i n s}}$ that characterizes the stress state of the body. When there exists an integer $N$ such that the previous representation holds for all subbodies of the considered body $B$, it is said that the body $B$ has a stress state of order $N$ in the sense of D'Alembert. The tensor measures $d \tau_{s, D}$ are naturally completely symmetric. They are called the s-th order (hyper)-stress tensors. In the literature, the only tensor measures which were considered are absolutely continuous with respect to the volume measure $d \mathscr{H}^{3}$, $d \tau_{k, D}=\tau_{k, D} d \mathscr{H}_{\mid D}^{3}$, with completely symmetric tensor densities. Moreover the densities are supposed to be smooth enough to be repeatedly integrated by parts.

It has also to be remarked that the only possible way for the densities $\tau_{s, D}$ to depend on the local form shape of $D$ is to be independent of $D$. Finally one deals with representations of the type:

$$
\mathfrak{S}^{\mathfrak{i n s}}(D, V)=-\sum_{k=0}^{N} \int_{D}\left(\nabla^{s} V\right) \mid \tau_{s} d \mathscr{H}^{3}
$$

This type of representation has first been considered by Green and Rivlin (see Green and Rivlin 1964a, b, c, 1965) who called the tensors $\tau_{s}$ the $s$-th order stresses.

\section{The Foundation of Higher Gradient Theories à la D'Alembert and à la Cauchy}

The mechanical postulation à la D'Alembert consists in assuming given a stress state $\mathfrak{S}^{\mathfrak{i n s}}$. Then the procedure is to rewrite it as the sum of a term of type $\int_{D} V \mid f d \mathscr{H}^{3}$ plus an expression similar to $\mathfrak{S}^{\mathfrak{f r o}}$. This deduction is simply obtained by a repeated application of the divergence theorem (possibly on submanifolds).

The mechanical postulation à la Cauchy uses a reverse procedure. It consists in assuming an expression for $\mathfrak{S}^{\mathfrak{f r o}}$ and rewriting it in a form similar to $\mathfrak{S}^{\mathfrak{i n} \mathfrak{s}}$. This is a more difficult procedure and, to be completed, it needs the following (quasi)-balance postulate: for every test field $V$, there exists a constant $K_{V}$ such that, for every subbody $D$

$$
\left|\mathfrak{S}^{\mathfrak{f r o}}(D, V)\right| \leq K_{V} \mathscr{H}^{3}(D) .
$$

The reader should notice that, when considering Cauchy continua and rigid virtual velocity fields $V$, the inequality (5) reduces to the quasi-balances of forces and moments done in the work (Noll 1990), but, as remarked in dell'Isola and Seppecher (1997), these quasibalances are not sufficient for obtaining a complete description of a stress state of order two or higher. While inequality (5) could seem a very weak assumption, it has been emphasized (see dell'Isola et al. 2016b) that it rules out some possible stress states, as, for instance, those occurring in continua including material surfaces or continua including interfaces with Laplace surface tension.

Even if often not explicitly stated, both procedures (Cauchy type and D'Alembert type) are always completed by using the postulate 
of work balance (or postulate of virtual work) and the aforementioned uniqueness result by L. Schwartz. This postulate states that the total mechanical interactions vanish. In formula:

$$
\mathfrak{S}^{\mathfrak{i n s}}+\mathfrak{S}^{\mathfrak{f} \mathfrak{r} \mathfrak{o}}=0
$$

For a presentation of the ideas inspiring this postulate, we refer to the papers (Germain 1973a, c; Salençon 2005) or to the historical works (Piola 1825, 1846 (translated in dell'Isola et al. 2014), Cosserat and Cosserat 1908, 1909). This equality, which holds for every admissible subbody and test field, dates back to the pioneering works of D'Alembert, Lagrange, and Piola (see Piola 1825, 1846; dell'Isola et al. 2014, 2015b) where it is shown that this principle is a generalization of Newton second law which is more suitable when dealing with more general systems than finite systems of material points.

Following D'Alembert, Lagrange, and Piola, one can find continuum mechanics by postulating a form for the work functional expressing internal interactions. Starting from this postulate, one can deduce, by means of a successive application of divergence theorem, the structure of the functionals expressing the contact interactions which can be exerted at the boundary of the considered body. Hence, this method starts from the notion of stress tensors (as dual of virtual displacements and their gradients) and deduces from it the concept and the structure of contact interactions by using the D'Alembert principle of virtual work. This principle is undoubtedly a great tool in mechanics. It has not been improved since its original first (and standard) formulation (differently to what was stated, e.g., in the paper Fried and Gurtin 2006). This is generally a position generally maintained in the literature (see, for instance, in Banfi et al. 2006).

In the approach à la D'Alembert, one assumes the principle of virtual work to be valid for every subbody of considered continuous body. This is done in all literature directly based on Lagrange's and Piola's works (see, e.g., Toupin 1962, 1964; Germain 1972, 1973a, b, c; Bleustein 1967; Casal 1961, 1972; Polizzotto 2007). An unduly restricted version of the Principle has been formulated in Truesdell and Toupin (1960), (pp. 595-600). For this reason many authors, at different times, rediscovered its correct and complete formulation.

At the beginning of the nineteenth century, Cauchy founded continuum mechanics by assuming that the surrounding material exerts on a part of a continuum a mechanical interaction limited to a surface density of contact forces concentrated on the dividing surface. Then, by assuming that these contact forces depend only on the normal of dividing surface and are balanced by some volume density of force (including inertia), he used the geometric properties of tetrahedrons to prove the existence of the so-called Cauchy stress tensor.

Within the context of higher gradient theories, the tetrahedron argument has to be treated with special attention (see dell'Isola et al. 2015b, 2016b; Auffray et al. 2015). In 1959, W. Noll (see Noll 1959) showed that the socalled Cauchy postulate, that is, the dependence of contact forces only on the normal of dividing surfaces, is equivalent to the seemingly weaker assumption of uniform boundedness of contact forces for all dividing surfaces. It has to be underlined that Cauchy postulate, despite its usual designation, is not a fundamental principle in the sense that balance of force or the principle of virtual work are but rather a constitutive assumption. Noll's result allows to point out the relationship between tetrahedron argument and measure theory (see, e.g., Del Piero 2014). However, one should pay special care not to forget, behind the technical hypothesis of uniform boundedness, the physical assumption that the contact forces depend only on the normal to the Cauchy cut. Actually the contact force per unit surface at any regular point of a Cauchy cut (in what is called face here) does not depend, in general, only on the orientation of such surface (i.e., only on its normal $n$ ). Several authors (among which Richard Toupin; see Toupin 1962, 1964) were aware of this fact, and in dell'Isola and Seppecher $(1995,1997)$ and dell'Isola et al. (2016b) some efforts have been 
done to generalize the tetrahedron construction in order to encompass theories of higher gradient continua. As explicitly remarked in dell'Isola and Seppecher (1995, 1997), Banfi et al. (2006), and Marzocchi and Musesti (2003), relevant mathematical difficulties are encountered when dealing with the double dependence of power functional $\mathfrak{S}^{\mathfrak{f r o}}(D, V)$ on velocity fields and on subbodies of the considered continuum. The efforts of many researchers (see, e.g., Banfi et al. 2006; Degiovanni et al. 2006; Marzocchi and Musesti 2003) are directed, with remarkable results, to the search of a generalized Schwartz representation theorem adapted to this context.

In De la pression ou tension dans un corps solide, Cauchy wrote (see Cauchy 1827 pp. 61-64) that "a small element experiences on its different faces and at each point of them a determined pressure or tension [...] which can depend on the orientation of the surface. This being set, $[\ldots]$ " and that "equilibrium should hold between inertial force and the forces to which are reduced all pressures and tensions exerted on the surface." In his proof, Cauchy applied the balance of forces to domains with a "volume very small, so that every dimension is an infinitesimal quantity of first order" the mass being "an infinitesimal quantity of the third order" and finally he stated that pressure and tension on a small face "experience, by moving from one point to another one on a face, infinitesimal variations of the first order." Clearly Cauchy accepted the following hypotheses: (i) contact interactions reduce to surface forces on the boundary and depend on its normal (ii) contact interactions are balanced by volume forces and (iii) contact interactions depend at least continuously on the position.

When accepting the form (3), one weakens the assumption (i), and when accepting the quasibalance postulate (5), one adapts assumption (ii) to the new context.

In fact the two methods can be reconciled. Their equivalence has already been explicitly established by Gabrio Piola (see dell'Isola et al. 2014) for stress states of order one. Much later the same equivalence has been proven for stress states of order two: these results have been obtained in the papers dell'Isola and Seppecher $(1995,1997)$ where the relationship between the concept of contact line force and surface double force was established by obtaining a representation formula relating the two concepts. Finally, a more general reconciliation which holds for stress states of order $n$ is provided in dell'Isola et al. (2015a).

\section{Some Remarks on Wave Propagation in Second Gradient Continua}

In this section we want to provide some quick and elementary remarks on wave propagation, focusing on the particular case of linearized isotropic second gradient continua.

Let us denote by $\mathbf{u}(\mathbf{X}, t)$ the displacement field, by $\mathbf{F}:=\nabla \chi$ the deformation gradient, and by $\varepsilon:=\left(\mathbf{F}^{T} \cdot \mathbf{F}-\mathbf{I}\right) / 2$ the classical GreenLagrange deformation tensor. If $\rho$ is the mass per unit volume of the considered continuum in its reference configuration, we can write the Lagrangian energy density of a second gradient continuum as

$$
\mathscr{E}=\frac{1}{2} \rho(\dot{\mathbf{u}})^{2}+\Psi(\boldsymbol{\varepsilon}, \nabla \boldsymbol{\varepsilon})
$$

where the deformation energy is denoted by $\Psi$, and we made explicit the fact that the objectivity requirements imply that it is only function of the Green tensor and its gradient (see Auffray et al. 2015).

In the absence of body forces, the equation of motion for a second gradient continuum reads (see, e.g., Germain 1973a)

$$
\operatorname{div}\left[\mathbf{F} \cdot\left(\frac{\partial \Psi}{\partial \boldsymbol{\varepsilon}}-\operatorname{div}\left(\frac{\partial \Psi}{\partial \nabla \boldsymbol{\varepsilon}}\right)\right)\right]=\rho \ddot{\mathbf{u}}
$$

Differentiating Eq. (6) with respect to time and using Eq. (7) and the symmetry properties of the tensors $\boldsymbol{\varepsilon}, \nabla \boldsymbol{\varepsilon}$, and $\nabla \mathbf{F}$, it can be shown that (see dell'Isola et al. 2012b) 


$$
\frac{\partial \mathscr{E}}{\partial t}+\operatorname{div}\left[-\dot{\mathbf{u}} \cdot \mathbf{F} \cdot\left(\frac{\partial \Psi}{\partial \boldsymbol{\varepsilon}}-\operatorname{div}\left(\frac{\partial \Psi}{\partial \nabla \boldsymbol{\varepsilon}}\right)\right)-\left((\nabla \dot{\mathbf{u}})^{T} \cdot \mathbf{F}\right): \frac{\partial \Psi}{\partial \nabla \boldsymbol{\varepsilon}}\right]=0 .
$$

which represents the Lagrangian form of energy balance for a second gradient continuum in the nonlinear case.

Linearizing in the neighborhood of a stressfree reference configuration, the gradient of placement $\mathbf{F}$ in Eq. (8) is substituted by the identity matrix and that the equation of motion and mechanical energy balance for a second gradient continuum reduce, respectively, to

$$
\begin{aligned}
& \operatorname{div}[\mathbf{S}-\operatorname{div} \mathbf{P}]=\rho \ddot{\mathbf{u}}, \quad \frac{\partial \mathscr{E}}{\partial t} \\
& +\operatorname{div}\left[-\dot{\mathbf{u}} \cdot(\mathbf{S}-\operatorname{div} \mathbf{P})-(\nabla \dot{\mathbf{u}})^{T}: \mathbf{P}\right]=0,
\end{aligned}
$$

where, following the nomenclature of Germain, $\mathbf{S}$ and $\mathbf{P}$ are the linearized Piola-Kirchhoff first and second gradient stress tensors, respectively.

It is well known that in the case of isotropic material $\mathbf{S}=2 \mu \mathbf{E}+\lambda(\operatorname{tr} \mathbf{E}) \mathbf{I}$, where $\mathbf{E}=$ $\left(\nabla \mathbf{u}+(\nabla \mathbf{u})^{T}\right) / 2$ is the linearized GreenLagrange deformation tensor and $\lambda$ and $\mu$ are the so-called Lamé coefficients. As for the hyperstress third-order tensor $\mathbf{P}$, it has been shown (see dell'Isola et al. 2009) that in the case of isotropic materials, it takes the following simplified form: (We define the transposition operations of a thirdorder tensor as $A_{i j k}^{T_{23}}=A_{i k j}$ and $A_{i j k}^{T_{12}}=A_{j i k}$ and the symbol $\otimes$ as the usual tensor product operation between two tensors of any order (e.g., $\left.\left.(A \otimes B)_{i j h k}=A_{i j} B_{h k}\right)\right)$

$$
\begin{aligned}
\mathbf{P}= & c_{2}\left[2 \mathbf{I} \otimes \operatorname{div} \mathbf{E}+(\mathbf{I} \otimes \nabla(\operatorname{tr} \mathbf{E}))^{T_{23}}+\nabla(t r \mathbf{E}) \otimes \mathbf{I}\right]+c_{3} \mathbf{I} \otimes \nabla(\operatorname{tr} \mathbf{E}) \\
& +2 c_{5}\left[(\mathbf{I} \otimes \operatorname{div} \mathbf{E})^{T_{23}}+\operatorname{div} \mathbf{E} \otimes \mathbf{I}\right]+2 c_{11} \nabla \mathbf{E}+4 c_{15}(\nabla \mathbf{E})^{T_{12}},
\end{aligned}
$$

where $c_{2}, c_{3}, c_{5}, c_{11}$, and $c_{15}$ are constants depending on the material properties of the considered second gradient continuum. Moreover, we define the following coefficients:

$\Lambda:=c_{3}+2\left(c_{5}+c_{15}\right)+4 c_{2}, M:=c_{11}+c_{15}+c_{5}$,

which parallel the first gradient Lamé coefficients $\lambda$ and $\mu$.

Considering a wave traveling in the considered second gradient continuum in the direction of the axis $x_{1}$ of an orthogonal reference system, and assuming that the displacement vector has three non-vanishing components depending only on the $x_{1}$ coordinate and on time, it is possible to obtain by straightforward computation (see dell'Isola et al. 2012b) the following dispersion relations:

$$
(\Lambda+2 M) k_{1}^{4}+(\lambda+2 \mu) k_{1}^{2}-\rho \omega^{2}=0,
$$

$$
\begin{aligned}
& M k_{2}^{4}+\mu k_{2}^{2}-\rho \omega^{2}=0, \\
& M k_{3}^{4}+\mu k_{3}^{2}-\rho \omega^{2}=0,
\end{aligned}
$$

where $\omega$ is the positive real frequency, $k_{1}$ is the wave number relative to the eigenvector $(1,0,0)$, and $k_{2}$ and $k_{3}$ are the wave numbers relative to the eigenvectors $(0,1,0)$ and $(0,0,1)$, respectively. Since we were considering an isotropic continuum, the last two transverse dispersion relations coincide.

\section{References}

Alibert J-J, Seppecher P, dell'Isola F (2003) Truss modular beams with deformation energy depending on higher displacement gradients. Math Mech Solids 8(1): 51-73

Auffray N, dell'Isola F, Eremeyev VA, Madeo A, Rosi G (2015) Analytical continuum mechanics la Hamilton- 
Piola least action principle for second gradient continua and capillary fluids. Math Mech Solids 20(4): 375-417

Banfi C, Marzocchi A, Musesti A (2006) On the principle of virtual powers in continuum mechanics. Ricerche di Matematica 55:299-310

Bleustein JL (1967) A note on the boundary conditions of Toupin's strain-gradient theory. Int J Solids Struct 3(6):1053-1057

Casal P (1961) La capillarité interne, Cahier du groupe Français de rhéologie, CNRS VI, 3, pp 31-37

Casal P (1972) La théorie du second gradient et la capillarité. C R Acad Sci Paris, t. 274, Série A 1571-1574

Cauchy AL (1827) De la pression ou tension dans un corps solide. Ex de Math 2:4256. Available in Gallica.bnf.fr

Chesnais C, Boutin C, Hans S (2012) Effects of the local resonance on the wave propagation in periodic frame structures: generalized Newtonian mechanics. J Acoust Soc Am 132(4), Pt. 2

Chesnais C, Boutin C, Hans C (2013) Wave propagation and non-local effects in periodic frame materials: generalized continuum mechanics. Math Mech Solids 20(8):929-958

Collin F, Chambon R, Charlier R (2006) A finite element method for poro mechanical modelling of geotechnical problems using local second gradient models. Int $\mathbf{J}$ Numer Methods Eng 65:1749-1772

Cosserat E, Cosserat F (1908) Note sur la théorie de l'action euclidienne. Gauthier-Villars, Paris

Cosserat E, Cosserat F (1909) Sur la Théorie des Corps Déformables. Herman, Paris

Coussy O (2004) Poromechanics. Wiley, Chichester

Degiovanni M, Marzocchi A, Musesti A (2006) Edgeforce densities and second-order powers. Annali di Matematica 185:81-103

dell'Isola F, Seppecher P (1995) The relationship between edge contact forces, double force and interstitial working allowed by the principle of virtual power. Comptes Rendus de l'Academie de Sciences - Serie IIb: Mecanique, Physique, Chimie, Astronomie, 321: 303-308

dell'Isola F, Seppecher P (1997) Edge contact forces and quasi-balanced power. Meccanica 32:33-52

dell'Isola F, Seppecher P (2011) Hypertractions and hyperstresses convey the same mechanical information. Continuum Mech Thermodyn (2010) 22:163-176. By Prof. Podio Guidugli and Prof. Vianello and some related papers on higher gradient theories. Continuum Mech Thermodyn 23(5):473-478

dell'Isola F, Sciarra G, Vidoli S (2009) Generalized Hooke's law for isotropic second gradient materials. Proc R Soc Lond Ser A Math Phys Eng Sci 465(2107):2177-2196

dell'Isola F, Seppecher P, Madeo A (2012a) How contact interactions may depend on the shape of Cauchy cuts in N-th gradient continua: approach á la D'Alembert. Zeitschrift fuer Angewandte Mathematik und Physik (ZAMP) 63(6):1119-1141
dell'Isola F, Madeo A, Placidi L (2012b) Linear plane wave propagation and normal transmission and reflection at discontinuity surfaces in second gradient 3D continua. ZAMM J Appl Math Mech/Zeitschrift fur Angewandte Mathematik und Mechanik 92(1): 52-71

dell'Isola F, Maier G, Perego U, Andreaus U, Esposito R, Forest S (2014) The complete works of Gabrio Piola: volume I. Advanced structured materials, vol 38. Springer International, Cham

dell'Isola F, Seppecher P, Della Corte A (2015a) The postulations á la D'Alembert and á la Cauchy for higher gradient continuum theories are equivalent: a review of existing results. In: Proceedings of the Royal Society A, vol 471, No 2183. The Royal Society, p 20150415

dell' Isola F, Andreaus U, Placidi L (2015b) At the origins and in the vanguard of peridynamics, non-local and higher-gradient continuum mechanics: an underestimated and still topical contribution of Gabrio Piola. Mech Math Solids 20(8):887-928

dell'Isola F, Steigmann D, Della Corte A (2016a) Synthesis of fibrous complex structures: designing microstructure to deliver targeted macroscale response. Appl Mech Rev 67(6):060804-060804-21

dell'Isola F, Madeo A, Seppecher P (2016b) Cauchy tetrahedron argument applied to higher contact interactions. Arch Ration Mech Anal 219(3): 1305-1341

Del Piero G (2014) Non-classical continua, pseudobalance, and the law of action and reaction. Math Mech Complex Syst 2(1):7110

Dunn JE (1986) Interstitial working and a non classical continuum thermodynamics. In: Serrin J (ed) New perspectives in thermodynamics. Springer, Berlin, pp 187-222

Dunn JE, Serrin J (1985) On the thermomechanics of interstitial working. Arch Ration Mech Anal 88(2): 95-133

Forest S (1998) Mechanics of generalized continua: construction by homogenization. J Phys IV Fr 8:Pr4 39Pr4 48

Forest S (2005) Generalized continua. In: Buschow KHJ, Cahn RW, Flemings MC, Ilschner B, Kramer EJ, Mahajan S (eds) Encyclopedia of materials: science and technology updates. Elsevier, Oxford, pp 1-7

Forest S (2006) Milieux continus généralisés et matériaux hétéérogènes. Les Presses de l'Ecole des Mines de Paris. isbn:2-911762-67-3

Forest S, Amestoy M, Cantournet S, Damamme G, Kruch S (2015) Mécanique des Milieux Continus, ECOLE DES MINES DE PARIS Année 2005-2006

Fosdick R (2011) On the principle of virtual power for arbitrary parts of a body. Continuum Mech Thermodyn 23:483-489

Fried E, Gurtin ME (2006) Tractions, balances, and boundary conditions for nonsimple materials with application to liquid flow at small length scales. Arch Ration Mech Anal 182:513-554 
Germain P (1972) Sur l'application de la méthode des puissances virtuelles en mécanique des milieux continus. C R Acad Sci Paris Série A-B 274: A1051-A1055

Germain P (1973a) La méthode des puissances virtuelles en mécanique des milieux continus. Première partie. Théorie du second gradient. J Mécanique 12: 235-274

Germain P (1973b) Cours de Mècanique des Milieux Continus, tome I. Masson, Paris

Germain P (1973c) The method of virtual power in continuum mechanics. Part 2: microstructure. SIAM J Appl Math 25:556-575

Green AE, Rivlin RS (1964a) Multipolar continuum mechanics. Arch Ration Mech Anal 17:113-147

Green AE, Rivlin RS (1964b) Simple force and stress multipoles. Arch Ration Mech Anal 16: 325-353

Green AE, Rivlin RS (1964c) On Cauchy's equations of motion. Z Angew Math Phys (ZAMP) 15: 290-292

Green AE, Rivlin RS (1965) Multipolar continuum mechanics: functional theory I. Proc R Soc Ser A 284:303-324

Marzocchi A, Musesti A (2003) Balanced virtual powers in continuum mechanics. Meccanica 38: 369-389

Mindlin RD (1962) Influence of couple-stresses on stress concentrations main features of cosserat theory are reviewed by lecturer and some recent solutions of the equations, for cases of stress concentration around small holes in elastic solids, are described. Exp Mech 3(1):1-7

Mindlin RD (1963) Complex representation of displacements and stresses in plane strain with couple-stresses. Appl Theory Funct Contin Mech (Proc Int Symp, Tbilisi), vol I (Mech Solids, Russian), pp 256-259 Izdat. Nauka", Moscow

Mindlin RD (1965a) Second gradient of strain and surface tension in linear elasticity. Int J Solids Struct 1(4): 417-438

Mindlin RD (1965b) Stress functions for a Cosserat continuum. Int J Solids Struct 1(3):265-271

Mindlin RD (1965c) On the equations of elastic materials with micro-structure. Int J Solids Struct 1(1): 73-78

Mindlin RD, Eshel NN (1968) On first strain-gradient theories in linear elasticity. Int J Solids Struct 4(1): 109-124

Mindlin RD, Tiersten HF (1962) Effects of couplestresses in linear elasticity. Arch Ration Mech Anal 11: 415-448

Noll W (1959) The foundations of classical mechanics in the light of recent advances in continuum mechanics. In: Proceeding of the Berkeley symposium on the axiomatic method, Amsterdam, pp 226-281

Noll W, Virga EG (1990) On edge interactions and surface tension. Arch Ration Mech Anal 111(1):1-31
Pideri C, Seppecher P (1997) A second gradient material resulting from the homogenization of an heterogeneous linear elastic medium. Contin Mech Thermodyn 9(5):241-257

Piola G (1825) Sull'applicazione de' principj della meccanica analitica del Lagrange ai principali problemi. Memoria di Gabrio Piola presentata al concorso del premio e coronata dall'I.R. Istituto di Scienze, ecc. nella solennita del giorno 4 ottobre 1824, Milano, Imp. Regia stamperia

Piola G (1846) Memoria intorno alle equazioni fondamentali del movimento di corpi qualsivogliono considerati secondo la naturale loro forma e costituzione, Modena, Tipi del R.D. Camera

Polizzotto C (2007) Strain-gradient elastic-plastic material models and assessment of the higher order boundary conditions. Eur J Mech A Solids 26(2): 189-211

Salençon J (2005) Mécanique des milieux continus: concepts généraux (vol 1). Editions Ecole Polytechnique

Schwartz L (1973) Théorie des Distributions. Hermann, Paris

Sciarra G, dell'Isola F, Coussy O (2007) Second gradient poromechanics. Int J Solids Struct 44(20): 6607-6629

Seppecher P, Alibert JJ, dell'Isola F (2011) Linear elastic trusses leading to continua with exotic mechanical. J Phys Conf Ser 319(1):012018

Steigmann DJ, Pipkin AC (1991) Equilibrium of elastic nets. Philos Trans R Soc Lond A: Math Phys Eng Sci 335(1639):419-454

Toupin RA (1962) Elastic materials with couple-stresses. Arch Rat Mech Anal 11:385-414

Toupin RA (1964) Theories of elasticity with couplestress. Arch Ration Mech Anal 17:85-112

Triantafyllidis N, Aifantis EC (1986) A gradient approach to localization of deformation. I. Hyperelastic materials. J Elast 16(3):225-237

Truesdell C, Noll W (1965) The non-linear field theories of mechanics. In: Fliigge's Encyclopedia of Physics, vol III/3. Springer, Berlin, pp 1-662

Truesdell C, Toupin RA (1960) The classical field theories. Handbuch der Physik III/1. Springer, Berlin 